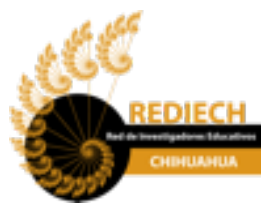

Red de Investigadores Educativos Chihuahua A.C. Chihuahua, México www.rediech.org

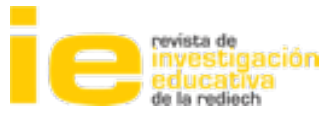

ISSN: 2007-4336

ISSN-e: $2448-8550$

http://www.rediech.org/ojs/2017/index.php/ie rie rediech/index

Karen Núñez-Valdés

José Alejandro Gronzález Campos

2019

\title{
Perfil de egreso doctoral: una propuesta desde el análisis documental y las expectativas de los doctorandos
}

IE Revista de Investigación Educativa de la REDIECH, 10(18), pp. 161-175.

http://dx.doi.org/10.33010/ie rie rediech.v10i18.604

\section{(c) (1) (9)}

Esta obra está bajo licencia internacional

Creative Commons Reconocimiento-NoComercial 4.0.

CC BY-NC 4.0 


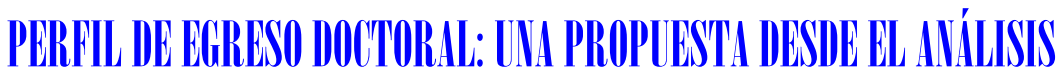 DOCINEATILL Y LLS EXPECTITIIIIS DE LOS DOCTTORIIIDOS
}

\author{
DOCTORLL GRIDLITE PROFHLE: I PROPOSSLL FROII DOCLINETTIRI \\ INLLLSIS IVI DOCTORAL STIDEVITS' EXPECTITIIOIS
}

\author{
NÚÑEZ-VALDÉS Karen \\ GONZÁLEZ CAMPOS José Alejandro
}

Recepción: enero 17 de 2019 | Aprobado para publicación: abril 1 de 2019

DOI: https://dx.doi.org/10.33010/ie_rie_rediech.v10il8.604

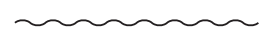

Resumen

En este artículo se presenta una discusión entre las expectativas de los estudiantes de doctorado con respecto a las competencias que debiesen adquirir tras su formación doctoral, la normativa europea, en específico los documentos emanados desde el Proceso de Bolonia y la normativa chilena, definida a través de las directrices elaboradas por la Comisión Nacional de Acreditación para la formación de posgrado. A partir de este diálogo se propone elaborar un perfil de egreso estándar para los programas de doctorado en educación a través de una metodología cuantitativa basada en el análisis de contenido. Como resultado se presenta un perfil de egreso estándar, basado en el modelo neozelandés, donde se contemplan las diferentes competencias que ha de adquirir un doctor en educación; con ello se pretende avanzar hacia la homogeneización

Karen Patricia Núñez Valdés. Profesora de historia, geografía y ciencias sociales de la Pontificia Universidad Católica de Valparaíso, Chile. Es licenciada en educación, licenciada en historia y magíster en educación mención evaluación educativa. Actualmente es candidata a doctor en política y gestión educativa de la Universidad de Playa Ancha, Chile. Se desempeña como asesora pedagógica en instituciones educativas y dicta cursos para los futuros docentes de historia y geografía de la Universidad de Playa Ancha. Participa activamente en congresos y seminarios de investigación. Su línea de investigación versa sobre temáticas como docencia universitaria, evaluación, gestión educativa y políticas públicas. Correo electrónico: k.nunez.valdes@gmail.com. ID: http://orcid.org/0000-0002-6641-6581.

José Alejandro González Campos. Académico-investigador de la Universidad de Playa Ancha, Chile. Es licenciado en educación, profesor de matemática y computación por la Universidad de Playa Ancha, magíster en estadística por la Pontificia Universidad Católica de Valparaíso y doctor en estadística por la Universidad Estatal de Campinas. Coordinador general de investigación de la Facultad de Ciencias Naturales y Exactas, coordinador de Laboratorio de Investigación y Experimentación de Saberes Matemáticos y Estadísticos de la Universidad de Playa Ancha. Posee más de diez publicaciones en los últimos dos años en revistas nacionales e internacionales. Correo electrónico: jgonzalez@upla.cl. ID: http://orcid.org/0000-0003-4610-6874. 
de los perfiles de egreso de este nivel universitario y contribuir a la evaluación de los perfiles de egreso ya existentes. Palabras clave: PERFILES DE EGRESO, COMPETENCIAS, ANÁLISIS DE
CONTENIDO.

\begin{abstract}
This article presents a discussion between the expectations of doctoral students related with the competences they should acquire after their doctoral training, the European regulations, specifically, the documents that come up from the Bologna Process and the Chilean regulations, defined through the guidelines developed by the National Accreditation Commission for postgraduate training. From this dialogue, it is proposed to develop a standard Graduate Profile for Doctoral programs in Education, through a quantitative methodology, based on content analysis. As a result, a standard Graduate Profile is presented, based on the New Zealand model, where the different competences that a Doctor of Education must acquire are contemplated, with it is intended to advance towards the homogenization of the Graduate Profiles of this university level and to contribute to the evaluation of existing Graduate Profiles.
\end{abstract}

Keywords: GRADUATE PROFILES, COMPETENCIES, CONTENT ANALYSIS.

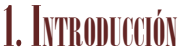

\section{La globalización, en su sentido más amplio, se ha traducido en} un intenso intercambio de información, de productos y de recursos, los que se han manifestado en la complejización y diferenciación de las sociedades, tanto al interior de ellas como en las relaciones entre estas (Elbaz y Helly, 2002). Este escenario planteó nuevos desafíos en términos de formación universitaria, pues cada vez más se hace imprescindible poseer profesionales altamente capacitados para hacer frente a las demandas que emergen, como consecuencias de la globalización. El doctorado, actividad formativa que otorga el grado académico más alto en una institución de educación superior, se constituye en la actualidad como un polo de formación de posgraduados altamente calificados, cuya importancia radica en la contribución que los doctores han de realizar a la sociedad a través de una investigación rigurosa y sistemática sobre una determinada temática y área del conocimiento. Cortina y González (cit. por Jiménez-Ramírez, 2017a, p. 134) afirman que "este segmento de la población activa se considera crucial en la producción, aplicación y difusión del conocimiento y, por tanto, clave de la mejora competitiva del país". En este sentido, dotar al país de doctores se transforma en una oportunidad de desarrollo estratégico, pues estos eventualmente contribuirán con la creación y difusión del conocimiento en diferentes áreas de interés y con ello al crecimiento económico, ya que "el cono-

162 cimiento ha pasado a ser considerado un bien económico y las universidades se han 
visto obligadas a canalizar las demandas de la nueva sociedad de la información" (Fernández y Wainerman, 2015, p. 156).

El desarrollo a nivel mundial de los programas de doctorado no ha sido homogéneo, pues cada región ha tomado sus propias decisiones en función de los objetivos planteados. Europa, por ejemplo, con el Proceso de Bolonia inició un proceso de reestructuración de la educación superior, incluyendo el nivel doctoral, donde se definieron las competencias que debía adquirir un aspirante a doctor en función de los requerimientos del espacio europeo. En Chile, por su parte, la creación de los doctorados es tardía, por lo que su desarrollo y regulación, en comparación a países desarrollados, e inclusive latinoamericanos, es menor (Vera, 2010). En Chile no existe:

[...] una ley que regule las formaciones de posgrado, por lo cual ellas pueden existir bajo cualquier estructura curricular, admitiendo cualquier formación previa y teniendo distintas duraciones. Desde este punto de vista, el sistema de posgrado chileno está fuertemente desregulado y la acreditación intenta actuar como poder regulador [Corvalán, Falabella y Rojas, 2011, p. 21].

Situación que se espera corregir con la implementación de la Ley $N^{\circ}$.21.091/2018 sobre educación superior, que incluirá nuevos mecanismos de regulación, destacándose la obligatoriedad de acreditación de los programas doctorales chilenos. Dentro de los criterios evaluados en los procesos de acreditación de estos programas se encuentra el denominado "Características y resultados del programa", siendo un elemento constitutivo de este el perfil de egreso. Un perfil de egreso es una declaración formal que realiza una institución de educación superior sobre los conocimientos, habilidades y aptitudes que deben alcanzar los graduados al finalizar un proceso de formación (Möller y Gómez, 2014). En virtud de esta situación, se propone elaborar un perfil de egreso, denominado estándar, para los programas doctorales del área de educación dictados en Chile con el fin de contribuir a la homogenización de los perfiles de egreso existentes en la oferta doctoral chilena, considerando lineamientos internacionales, nacionales y las expectativas de los estudiantes de doctorado chilenos, triada que se constituirá en el insumo de esta propuesta.

\subsection{Perfil de egresso}

Las universidades han comenzado a ser cada vez más exigidas, especialmente porque la sociedad en el estado actual de globalización reclama la calidad de sus egresados para su posterior inserción laboral. En este contexto, la formación universitaria ha transitado en las últimas décadas hacia modelos de formación por competencias o por resultados de aprendizaje, según cada institución y/o programa, intentando operacionalizar estos modelos en la definición de perfiles de egreso (Möller y Gómez, 2014). En función de la implementación de estos, los programas de pregrado y posgrado ofrecidos en el sistema universitario deben contar con perfiles de egreso, cuyo fin es que los aspirantes y el público general conozcan el compromiso de la universidad para con este futuro profesional. Hawes y Troncoso $(2012$, p. 2) señalan que: 
El Perfil de Egreso se concibe como una declaración formal que hace la institución frente a la sociedad y frente a sí misma, en la cual compromete la formación de una identidad profesional dada, señalando con claridad los compromisos formativos que contrae y que constituyen el carácter identitario de la profesión en el marco de la institución, a la vez que especifica los principales ámbitos de realización de la profesión y sus competencias clave asociadas.

En tanto para Möller y Gómez (2014, p. 25), el "perfil de egreso (como definición identitaria y como compromiso formativo), se concibe como un instrumento que dota de sentido a los programas de formación, por lo que resulta fundamental que los perfiles incluyan mecanismos para evaluar su cumplimiento". En esta misma línea, Knust y Gómez (2009) señalan que el perfil de egreso, al contener las competencias que se han de desarrollar en un determinado proceso formativo, se constituye en una herramienta útil para la evaluación del desempeño de los futuros profesionales.

De este modo, un perfil de egreso puede ser entendido como la definición que realiza una institución de educación superior sobre el tipo de profesional que espera formar, declarándose en él las competencias que un individuo ha de adquirir al finalizar un proceso formativo, tanto propias al ámbito en cual se desempeñará, como aquellas transversales al desarrollo profesional. El perfil de egreso se convierte así en una herramienta para evaluar la adquisición de competencias de un determinado profesional.

En Chile, la elaboración de los perfiles de egreso de los programas de pregrado se ha llevado a cabo a través de diferentes instancias, siendo una de las más frecuentes "la organizaron de equipos de trabajo conformados por representantes de las áreas académica, profesional y, muchas veces, también de empleadores o usuarios" (Comisión Nacional de Acreditación y Ministerio de Educación, 2007, p. 41), quienes definen los resultados de aprendizaje esperados para una determinada carrera 0 programa. De este modo, para la elaboración de los perfiles de egreso de pregrado, las instituciones de educación superior cuentan con la asistencia de la Comisión Nacional de Acreditación, quien, a través de distintos documentos, orienta su creación y posterior declaración pública.

En el caso de los programas de posgrados, y en específico de los doctorados, se presume que la guía a utilizar para la formulación del perfil de egreso es la misma que se utiliza en los perfiles de pregrado, ya que no existe un documento exclusivo para esta etapa de formación. La Comisión Nacional de Acreditación, a partir de la "Guía para la elaboración del informe de autoevaluación" de los programas de posgrado, orienta la creación de los perfiles de egreso a través de la declaración de los aspectos que evaluará, a saber:

[...] claridad en la definición de los objetivos y el perfil de egreso, objetivos y perfil de egreso coherentes con el carácter del programa, perfil de egreso específico y claro respecto a las actividades académicas del programa, mecanismos de revisión y difusión del perfil de egreso, consistencia de las líneas de investigación desarrolladas, con el carácter, objetivos y perfil de egreso y continuidad en el tiempo de las líneas de inves164 tigación [Comisión Nacional de Acreditación, 2013, p. 6]. 
Asimismo, para verificar la pertinencia del perfil de egreso de cada programa, la Comisión Nacional de Acreditación (2013) sugiere el uso de preguntas orientativas, entre ellas: ¿existen mecanismos definidos para la revisión del perfil?, ¿estos consideran su validación externa e interna?, ¿cuál es la periodicidad con la que este es revisado?, entre otras.

\subsection{Eurropa y el nivel dootorial}

El siglo Xx supuso para el espacio europeo una serie de desafíos en términos de formación universitaria, ya que la diversidad relevaba una serie de incompatibilidades que imposibilitaban la movilidad de los estudiantes y el reconocimiento de los diferentes grados académicos, repercutiendo esto directamente en la cooperación entre países (Balaban y Wright, 2014). En este contexto emerge el Proceso de Bolonia, cuyo objetivo inicial era implementar un sistema universitario más compatible, con dos ciclos de enseñanza, a saber, el grado y el posgrado (magíster y doctorado). Este proceso permitió reconsiderar el sistema universitario europeo y realizar una serie de cambios en él. A pesar de estos cambios, la educación doctoral no fue parte del debate inicial, por lo que los estudiantes de doctorado formaron el año 2002 el European Council of Doctoral Candidates and Junior Researchers con la finalidad de incluir este nivel universitario en el debate. Según Balaban y Wright (2014), esta iniciativa, junto con otras estrategias, permitió el inicio de las discusiones sobre el doctorado, específicamente en torno a su identidad y al tipo de formación que se estaba entregando. Jiménez-Ramírez (2017b) señala que, con la Declaración de Berlín del año 2003, la estructura europea de educación superior se completó con un tercer ciclo, el nivel doctoral, dejando este de ser parte del posgrado, el que se había constituido en los inicios del Proceso de Bolonia como el segundo ciclo de formación.

De este modo, Europa, a partir de la Declaración de Berlín, comienza a: "Destacar la importancia de la investigación y la formación en la investigación, junto con la promoción de la interdisciplinariedad para mantener y mejorar la calidad de la educación superior y para hacer la educación superior en Europa más competitiva en general" (Declaración de Berlín, 2003, en Annet et al., 2012, p. 249).

Asimismo, se llamó a incrementar la movilidad de los doctores y se animó a las instituciones a cooperar con los estudiantes de doctorado, quienes representaban la nueva generación de investigadores europeos. En síntesis, la Declaración de Berlín colocó de manifiesto que el doctorado se constituye como la primera fase de la carrera investigadora, siendo "el tercer ciclo de estudios universitarios oficiales, conducentes a la adquisición de competencias y habilidades relacionadas con la investigación científica de calidad" (Horgué, 2012, p. 373).

El año 2005, en Salzburgo se realizó la publicación de los diez principios básicos del nivel doctoral, resaltándose la importancia de la investigación original y el compromiso que las instituciones de educación superior debían asumir para con este nivel. El mismo año, en la Declaración de Bergen, se manifestó "la importancia de establecer una formación doctoral para la investigación, ensalzando a los doctorandos como investigadores imprescindibles para fortalecer un conocimiento científico" (Jiménez-Ramírez, 2017a, p. 125). Esta declaración se enmarca en una sociedad 
compleja y globalizada, en cambio constante, que requiere de avances que permitan el crecimiento y desarrollo de cada uno de los países que componen el espacio europeo. Es a partir de estas declaraciones que Europa perfila la formación de nivel doctoral alineando esta con las necesidades de una sociedad en constante transformación, que requiere de un conocimiento actualizado y capaz de asumir nuevos desafíos. En consecuencia, se inicia la definición de las competencias básicas que debía poseer un futuro doctor, cuya importancia radicaba en que estas le permitirían desenvolverse tanto en el área del conocimiento en la que investiga, así como impactar a su entorno a través de la transferencia del conocimiento.

Balaban y Wright (2014) señalan que después del año 2005, el proceso pasó de la conceptualización a la implementación, naciendo el año 2008 el Consejo para la Educación de Doctorado, lo que permitió el desarrollo de un plan de trabajo detallado, la creación de una serie de documentos para la modernización de las universidades y distintos tipos de financiamiento para proyectos europeos conjuntos. Gracias a esta serie de acciones, la universidad del siglo XXI asignó un lugar de gran relevancia a la investigación, constituyéndose esta como una marca de fábrica. En términos de competencias, Nebot (2009) señala que, tras el proceso iniciado en Bolonia, se han definido las competencias que han de adquirir los futuros doctores. Estas se han clasificado en disciplinares, metodológicas, instrumentales y genéricas o transferibles. Para este autor:

La formación en las competencias disciplinares y metodológicas se obtiene principalmente en el periodo de formación del programa de doctorado; las habilidades, en la realización de la tesis doctoral, pero la formación en competencias transferibles ha de asumirse institucionalmente y, donde existen, se organiza a través de las escuelas doctorales [Nebot, 2009, p. 15].

En definitiva, con el Proceso de Bolonia, y posteriormente con el Proyecto Tuning, se planteó una nueva forma de mirar la enseñanza de pregrado y posgrado, donde se enfatiza el desarrollo de competencias específicas y genéricas en una determinada área del conocimiento (Villarroel y Bruna, 2014).

\subsection{El doctoriado en (hile}

En Chile, el posgrado, especificativamente el doctorado, es relativamente reciente. Vera (2010) señala que el desarrollo tardío de este nivel radica en que el interés del Estado chileno durante un largo periodo estuvo centrado en la formación de licenciados (pregrado), lo que trajo como consecuencia el descuido del posgrado.

El año 1947 se creó el primer programa doctoral en una institución de educación superior chilena. Según Baeza (2017), este primer programa permitió el nacimiento de la oferta de programas doctorales en territorio chileno, consolidándose en primera instancia las áreas disciplinarias tradicionales, para luego avanzar hacia áreas como ciencias sociales y educación. Espinoza y González (2009) resaltan que esto obedece a la tendencia mundial a incrementar los estudios de cuarto nivel, lo que se 
ha evidenciado en la diversificación de la oferta y en el aumento de la matrícula en Chile, característica transversal de la educación superior.

Este panorama cambió drásticamente como consecuencia de la globalización, ya que sus implicaciones han impactado directamente la educación de cuarto nivel, pues se ha hecho cada vez más necesario "contar con académicos, profesionales y técnicos de excelencia, que adquieran las competencias para el desarrollo científico y tecnológico, la innovación y el emprendimiento" (González y Jiménez, 2014, p. 133). Con el fin de incentivar a los profesionales chilenos a cursar estudios doctorales, el Estado de Chile creó fondos de financiamiento para permitir el acceso a este nivel. Estas iniciativas han sido parte de una serie de políticas públicas guiadas por organismos supranacionales, como el Banco Mundial y la Organización para la Cooperación y el Desarrollo Económicos (OCDE), los cuales relevan el valor que posee el conocimiento y la educación en una sociedad globalizada (Chiappa y Muñoz, 2015). Estos incentivos se materializaron en una primera instancia con la Beca Presidente de la República y más tarde con el programa Becas Chile, financiamiento otorgado en función del mérito de los aspirantes, siendo requisito para su postulación estar matriculado en un programa de doctorado acreditado. En la actualidad, la oferta de programas doctorales ha crecido notoriamente en comparación a décadas precedentes. En el caso de los doctorados del área de educación, para el año 2018 existen 20 programas vigentes, 7 de ellos acreditados, teniendo en su totalidad 321 estudiantes matriculados (Ministerio de Educación, 2018).

\section{APIVTRE METHODOĹńGico}

En adelante, se presenta la metodología empleada para el desarrollo de esta investigación, la cual ha sido dividida en dos subsecciones: objetivos, técnica de recolección $\mathrm{y}$ análisis de datos.

\subsection{Objetivos}

En este estudio, vinculado a los perfiles de egreso de los doctorados del área de educación, se proponen los siguientes objetivos de investigación: 1) identificar las competencias que componen los perfiles de egreso de los doctorados dictados en el Espacio Común Europeo; 2) determinar los elementos exigidos por la Comisión Nacional de Acreditación de los programas de doctorado dictados en Chile para los perfiles de egreso; 3 ) establecer las expectativas de los doctorandos en educación sobre las competencias que esperan adquirir al obtener este grado académico; y, 4) elaborar un perfil de egreso estándar para los programas de doctorado del área de educación chilenos a partir de la triada documentos internacionales, nacionales y expectativas de los doctorandos.

\subsection{Téconicia de recolección y annilisisis de dâtos}

Atendiendo a los objetivos antes expuestos, se ha optado por una metodología de tipo cuantitativa. La técnica de recolección de datos a utilizar será el análisis de contenido 
de tipo cuantitativo. Núñez Valdés (2017) señala que este parte del supuesto de que el hablar y el escribir representan una forma de conducta social; por lo tanto, lo que se dice 0 se escribe revela las intenciones, actitudes, interpretaciones, conocimientos y supuestos sobre el entorno. El objetivo de esta técnica es lograr la emergencia del sentido latente de las prácticas sociales y cognitivas que instrumentalmente recurren a la comunicación, pretendiéndose indagar en aquello que está en el texto. Berelson señala que "el análisis de contenido es una técnica de investigación objetiva, sistemática y cuantitativa del contenido manifiesto de la comunicación" (cit. por Ander-Egg, 1993, p. 32). En esta investigación se realizó un análisis de contenido con base gramatical, donde la unidad de análisis fueron las frases y los párrafos. Además, es de tipo descriptivo, ya que tiene por objeto la identificación y catalogación de la realidad empírica de los textos y/o documentos mediante la definición de categorías o la clasificación de sus elementos (Piñuel, 2002). Para este estudio se han definido categorías de materia o contenido, las que se refieren a las competencias que ha de poseer un doctor en educación tras la obtención de este grado académico. El análisis realizado es de tipo transversal, ya que se ha seleccionado una muestra de corpus textuales diferentes, susceptibles de ser analizadas en un mismo momento histórico (Piñuel, 2002).

Se definieron tres grupos muestrales. El primero de ellos, una serie de documentos elaborados desde el Proceso de Bolonia, iniciado en Europa el año 1999, con el fin de obtener información sobre la normativa europea sobre los doctorados, las competencias que entregan estos y sus correspondientes perfiles de egreso. El segundo grupo muestral corresponde a los documentos emanados desde la Comisión Nacional de Acreditación (Chile) que regulan los programas doctorales chilenos y contienen directrices sobre los perfiles de egreso de este nivel universitario. El último grupo muestral se refiere a las opiniones de los doctorandos sobre sus expectativas al finalizar el programa de doctorado que cursan. El muestreo realizado fue de tipo aleatorio. Considerando el número total de estudiantes matriculados al año 2018, determinándose con la fórmula de Namakforoosh (2000) que el tamaño de la muestra sería de 94 doctorandos (error de estimación de un 5\% y un nivel de confianza de un 95\%). Las respuestas de los doctorandos fueron procesadas en el software R-Project en su versión 3.5.1.

Con el objetivo de identificar y catalogar la realidad empírica de los documentos, se definieron a priori categorías de análisis, las que se presentan en la tabla 1.

Tabla 1. Grupos muestrales y sus respectivas categorías de análisis

\begin{tabular}{ll}
\hline Grupo muestral & Categorías analíticas \\
\hline Documentos elaborados desde el & Competencias que ha de poseer un doctor. \\
Proceso de Bolonia a la actualidad. & Demostración del dominio de competencias de un doctor. \\
\hline Documentos emanados desde la & $\begin{array}{l}\text { Dimensión en la que se encuentra el perfil de egreso en } \\
\text { los procesos de acreditación. }\end{array}$ \\
$\begin{array}{l}\text { Comisión Nacional de Acreditación } \\
\text { (Chile). }\end{array}$ & $\begin{array}{l}\text { Estándar más alto en términos de evaluación de perfil de } \\
\text { egreso. }\end{array}$ \\
\hline Opinión de los doctorandos. & $\begin{array}{l}\text { Competencias que esperan alcanzar los doctorandos al } \\
\text { finalizar su formación. }\end{array}$ \\
\hline Fuente: Elaboración personal. & \\
\hline
\end{tabular}




\section{Ressutinos}

En adelante se presentan los resultados obtenidos tras el análisis de contenido realizado a cada uno de los grupos muestrales de esta investigación. En términos del grupo muestral número uno, denominado "Documentos elaborados desde el Proceso de Bolonia a la actualidad", se llevó a cabo una búsqueda exhaustiva de todos aquellos textos que fueron parte de las diferentes instancias donde se discutió el futuro de la educación superior europea, especialmente en lo referido al doctorado. Las categorías determinadas a priori permitieron la síntesis de la información recolectada, identificándose así el grupo de competencias que debía adquirir un doctorando al finalizar su formación. En la tabla 2 se da cuenta de estas competencias, con su respectiva descripción:

El grupo muestral número dos, denominado "documentos emanados desde la Comisión Nacional de Acreditación (Chile)" se analizó bajo la categoría analítica "Dimensiones propuestas por la Comisión Nacional de Acreditación", revisándose los documentos donde se operacionalizan los criterios de evaluación. En términos de los criterios referidos a los perfiles de egreso se utilizó el tramo que determina que el perfil cumple con todos los requerimientos de la normativa nacional vigente, ya que este es el estándar a alcanzar. En la tabla 3 se presenta el análisis realizado y se ha agregado el concepto del perfil de egreso como de criterio de evaluación.

Cabe señalar que en el análisis realizado al grupo muestral número dos queda en evidencia que la normativa chilena no explicita las competencias que debiese adquirir un futuro doctor, sino que pide a la institución que imparte un determinado programa de doctorado que explicite los conocimientos y habilidades que ha de entregar a sus graduados, por lo que cada institución posee libertad para establecer el tipo de competencias que espera que sus doctorandos alcancen al finalizar el proceso formativo.

En lo referido al grupo muestral número tres "Opinión de los doctorandos" se utilizó como técnica la cuantificación de la frecuencia de las diez palabras más utilizadas en las respuestas sobre las expectativas que tenían los futuros doctores sobre las competencias que deseaban adquirir al finalizar el programa que cursaban. Se utilizaron para graficar estos resultados las aplicaciones Wordcloud y ggplot, ambas de carácter libre. En la figura uno se evidencian las competencias que esperan adquirir los doctorandos, las cuales fueron agrupadas según su frecuencia.

Luego de determinadas estas competencias se elaboró la tabla 4, donde se explica cada una de estas con el fin de establecer, a través de la literatura, las características de las competencias que se esperan adquirir.

Tras la triangulación del análisis documental y el análisis de las opiniones de los doctorandos se ha elaborado un perfil de egreso, denominado en adelante perfil de egreso estándar para programas de doctorado en educación. Este perfil se ha escrito bajo el modelo presentado por Spronken, Brown y Mirosa (2018), quienes utilizan el modelo neozelandés para la declaración de los perfiles de egreso. Este modelo se caracteriza por tres elementos, a saber: 1) la descripción del graduado; 2) las competencias que ha de adquirir este en su formación; y, 3) los atributos o competencias genéricas que desarrollará. El perfil estándar presentado se ha inspirado en los tipos de competencias que fueron determinadas en el Espacio Europeo, considerando que 
Tabla 2. Competencias que ha de poseer un doctor según el Marco Común Europeo

\begin{tabular}{lll}
\hline $\begin{array}{l}\text { Competencias } \\
\text { de un doctor } \\
\text { (Nebot, 2009) }\end{array}$ & $\begin{array}{l}\text { Lo que ha de } \\
\text { demostrar un } \\
\text { doctor (Nebot, } \\
\text { 2009) }\end{array}$ \\
\hline
\end{tabular}

\begin{tabular}{|c|c|c|}
\hline Disciplinares & $\begin{array}{l}\text { Demostrar com- } \\
\text { prensión en un } \\
\text { campo de estudio. }\end{array}$ & $\begin{array}{l}\text { Según Alliaud y Vezub (2015, p. } 115) \text {, el campo de estudio se carac- } \\
\text { teriza por los siguientes temas de estudio: } \\
\text { - Saberes disciplinares (contenido a enseñar). } \\
\text { - Desarrollo cultural, social y psicológico de los/las estudiantes. } \\
\text { - Currículo. } \\
\text { - Didáctica. } \\
\text { - Evaluación. } \\
\text { - Gestión escolar. } \\
\text { - Tecnologías de la información. } \\
\text { - Estrategias para educar en la diversidad. } \\
\text { - Conocimiento de los contextos educativos. }\end{array}$ \\
\hline
\end{tabular}

Ary et al. (2018) señalan que los métodos de investigación en educación son:

- Método de investigación cualitativo: caracterizado por la búsqueda

Demostrar domi-

Metodológicas nio de métodos de investigación. de una comprensión más profunda de un fenómeno, centrado en la imagen total en vez de dividirla en variables y analizarlas numéricamente.

- Método de investigación cuantitativo: fundamentado en la medición de las características de los fenómenos sociales, expresando sus análisis en la relación entre las variables estudiadas; se tiende a generalizar resultados.

\begin{tabular}{|c|c|c|}
\hline Instrumentales & $\begin{array}{l}\text { Demostrar domi- } \\
\text { nio de técnicas de } \\
\text { investigación. }\end{array}$ & $\begin{array}{l}\text { Diseños de investigación cualitativa. } \\
\text { Padgett (2016) da cuenta de los seis enfoques primarios en la investi- } \\
\text { gación cualitativa, a saber: } \\
\text { - Etnografía. } \\
\text { - Ground Theory. } \\
\text { - Estudio de caso. } \\
\text { - Enfoque narrativo. } \\
\text { - Análisis fenomenológico. } \\
\text { - Investigación-acción. } \\
\text { Diseños de investigación cuantitativa: } \\
\text { Padua (2018) señala que en términos de investigación cuantitativa se } \\
\text { distinguen tres diseños: } \\
\text { - Estudios exploratorios. } \\
\text { - Estudios descriptivos. } \\
\text { - Estudios explicativos. }\end{array}$ \\
\hline $\begin{array}{l}\text { Genéricas o } \\
\text { transferibles }\end{array}$ & & $\begin{array}{l}\text { Las competencias genéricas, comunes a todas las profesiones, } \\
\text { incluyen elementos de orden cognitivo y de orden motivacional, y se } \\
\text { expresan a través de las denominadas: } \\
\text { - Competencias instrumentales, de orden metodológico o de } \\
\text { procedimiento, tales como la capacidad de análisis y síntesis, de } \\
\text { organización y planificación y de gestión de información. } \\
\text { - Competencias personales, tales como la capacidad para el } \\
\text { trabajo en equipo, la habilidad para el manejo de las relaciones } \\
\text { interpersonales, el compromiso ético. } \\
\text { - Competencias sistémicas, que se manifiestan en el aprendizaje } \\
\text { autónomo, la adaptación a nuevas situaciones, la creatividad y el } \\
\text { liderazgo, entre otras (Maura y Tirados, 2008, p. 193). }\end{array}$ \\
\hline
\end{tabular}

170 Fuente: Elaboración personal. 
Tabla 3. El perfil de egreso y su evaluación según la Comisión Nacional de Acreditación

\begin{tabular}{|c|c|c|}
\hline $\begin{array}{l}\text { Dimensión } \\
\text { (Comisión Na- } \\
\text { cional de Acredi- } \\
\text { tación, 2013) }\end{array}$ & $\begin{array}{l}\text { Cuarto tramo. Acreditación de un progra- } \\
\text { ma por el máximo de años establecidos }(8 \\
\text { a } 10 \text { años) (Comisión Nacional de Acredi- } \\
\text { tación, } 2016, \text { p. 10) }\end{array}$ & $\begin{array}{l}\text { Perfil de egreso como criterio de eva- } \\
\text { luación (según diversos autores) }\end{array}$ \\
\hline \multirow[t]{2}{*}{$\begin{array}{l}\text { Carácter, obje- } \\
\text { tivos y perfil de } \\
\text { egreso }\end{array}$} & $\begin{array}{l}\text { Subcriterio 1. Objetivos y perfil de egreso } \\
\text { El programa cuenta con objetivos y perfil de } \\
\text { egreso explícitos, claramente definidos, } \\
\text { consistentes entre sí y coherentes con } \\
\text { el carácter académico del programa y la } \\
\text { misión de la institución. } \\
\text { El perfil de egreso establece con claridad } \\
\text { los conocimientos y las habilidades de los } \\
\text { graduados para desarrollar investigación } \\
\text { original y autónoma. }\end{array}$ & $\begin{array}{l}\text { Verdejo (2008) señala que el perfil de } \\
\text { egreso es aquel que contiene el con- } \\
\text { junto de competencias que supone un } \\
\text { estudiante pondrá al servicio de su } \\
\text { profesión. Desde esta perspectiva, la } \\
\text { competencia se demuestra en la acción } \\
\text { o ejecución. Para poder evaluar el grado } \\
\text { de dominio de la competencia se cuenta } \\
\text { con el perfil de egreso. }\end{array}$ \\
\hline & $\begin{array}{l}\text { Subcriterio 2. Actualización del perfil de } \\
\text { egreso } \\
\text { El programa dispone de procedimientos } \\
\text { de actualización y validación del perfil } \\
\text { de egreso, los que han sido aplicados } \\
\text { sistemáticamente. }\end{array}$ & $\begin{array}{l}\text { Para Knust y Gómez (2009), el perfil } \\
\text { de egreso, al dar cuenta de las compe- } \\
\text { tencias que han de ser adquiridas en } \\
\text { un determinado programa formativo, } \\
\text { este se constituye en una herramienta } \\
\text { de evaluación del desempeño de los } \\
\text { futuros profesionales. }\end{array}$ \\
\hline
\end{tabular}

Fuente: Elaboración personal.

Tabla 4. Competencias que ha de adquirir un doctor según las expectativas de los doctorandos chilenos

\section{Referencia de los doctorandos sobre lo que esperan de su formación}

Investigación

Análisis

Escritura académica

Conocimiento

Autonomía

\section{Descripción de la competencia (según diversos autores)}

Dentro de las competencias sobre el proceso de la investigación destacan la capacidad para teorizar y construir modelos y las competencias de escritura científica, así como la habilidad relacional del investigador con los sujetos de investigación, la gestión de datos (Rivas, 2011, p. 36).

El pensamiento analítico se define como la capacidad de distinguir y separar las partes de un todo hasta llegar a conocer sus principios o elementos. Este tipo de pensamiento se destaca por el detalle, la precisión, la enumeración y la diferencia (Villa y Poblete, 2007).

Son tres los aspectos fundamentales que deben desarrollarse para dominar esta competencia. Aprender las técnicas de citación científica, aprender el estilo de redacción científico, aprender y conocer la estructura de los trabajos de investigación científicos más comunes: la de la tesis de investigación y la del artículo científico (Rivas, 2011, p. 48).

Elaborar un marco conceptual: abordar un problema de investigación supone describir el marco de referencia sobre el problema de investigación en el mundo y en el país que se lleva a cabo la investigación (Rivas, 2011, p. 40).

Según Collingwood (cit. por Scott, 2001, p. 55), esta se refiere a la condición de ser la autoridad propia, haciendo afirmaciones o tomando acción por iniciativa propia y no porque esas afirmaciones o acciones sean autorizadas o prescritas por cualquier otro. 
Fig. 1. Competencias que esperan alcanzar los doctorandos al finalizar su formación.

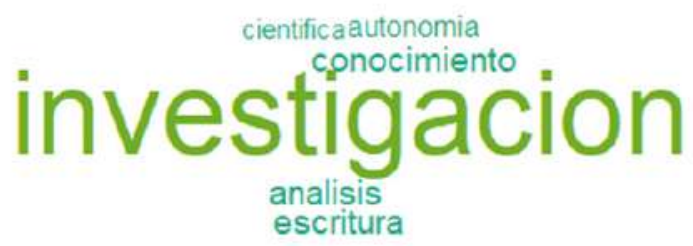

Fuente: Elaboración personal.

la normativa chilena no hace alusión a estas. A continuación se presenta la propuesta desarrollada.

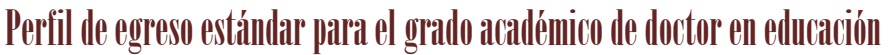

Un graduado con el grado de doctor en educación demostrará habilidades altamente desarrolladas en la definición, gestión y comunicación de investigaciones originales en un área especializada.

\section{Defininión del (rimpo de estudio}

Un doctor en educación realizará una contribución significativa al conocimiento en su campo de especialización, por lo que, al completar sus estudios, será capaz de:

- Realizar investigación de la más alta calidad que aporte al conocimiento disciplinar tanto a nivel nacional como internacional.

- Desarrollar su línea de investigación con el fin de contribuir a la comunidad científica a la cual pertenece.

- Dominar tanto metodologías cuantitativas como cualitativas de tal forma de realizar la elección de esta en función de los objetivos propuestos para una determinada investigación.

- Evaluar y aplicar las técnicas de investigación cuantitativas y cualitativas con el fin seleccionar aquella que aborde con mayor comprensión los fenómenos a investigar.

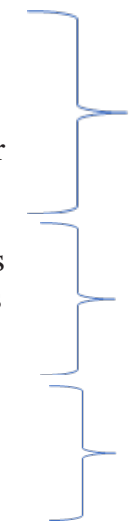

Competencias disciplinares

Competencias metodológicas

Competencias instrumentales

Los doctores en educación poseerán las siguientes competencias transferibles: ya que cada institución deberá definir qué competencias genéricas espera

- Pensamiento crítico.

- Ética.

- Trabajo en equipo.

- Liderazgo. desarrollar en sus doctorandos.

Se sugiere que cada institución defina qué entiende por cada una de las competencias genéricas con las que se compromete. 


\section{Conculusionses}

El doctorado, grado académico más alto otorgado por una universidad, ha adquirido gran relevancia en una sociedad cada vez más compleja, producto de la globalización, donde el éxito económico es preponderante para los diferentes países. Este nivel educativo comenzó a posicionarse como fundamental para alcanzar las metas de crecimiento y desarrollo económico, pues son los futuros doctores los llamados a hacer frente a las demandas de la sociedad del conocimiento a través de investigaciones originales que permitan solucionar los problemas que emergen producto de los constantes cambios en los que se vive.

Es por esta razón que en Europa se inició en la década de los noventa una convergencia en torno a la educación superior; no obstante, el nivel doctoral quedó rezagado hasta la década siguiente, donde se iniciaron las discusiones sobre lo que debería ser un doctor y cuáles serían las competencias que debía poseer este para fortalecer la investigación y los intercambios tanto de personas como de ideas en un espacio geográfico diverso. En Chile este proceso inició recientemente, específicamente a partir de la consolidación de la acreditación de los programas de educación universitaria, lo que ha colocado de manifiesto el carácter laxo que existe en torno a los programas doctorales y la necesidad de regular estos en función de las demandas tanto nacionales como internacionales.

No es de extrañar que muchos de los programas de doctorado en educación dictados en Chile no posean una certificación de calidad, en específico el $65 \%$ de ellos, lo que a la postre significa que no existe una regulación sobre esta, lo que resulta preocupante considerando que con la aprobación de la Ley $\mathrm{N}^{\circ} 21.091 / 2018$ desde el año 2020 no podrán funcionar programas que no estén acreditados.

Por tal motivo, esta investigación ha indagado tanto en la normativa internacional, específicamente la europea y la nacional, emanada desde la Comisión Nacional de Acreditación, con el fin de establecer parámetros que permitan a las instituciones de educación superior desarrollar perfiles de egreso alineados a programas internacionales reconocidos por su excelencia. De igual forma, se consideró la opinión de los estudiantes de doctorado con el fin de identificar las competencias que esperan adquirir al finalizar el programa que cursan, siendo relevante que lo expresado por los doctorandos se encuentra alineado con las propuestas europeas en cuanto a las competencias de un doctor.

En este sentido, la propuesta realizada, denominada perfil de egreso estándar, pretende contribuir a la generación de perfiles de egreso doctorales tanto en el territorio chileno como en otros países latinoamericanos, pues se constituye como un insumo para los gestores universitarios. En definitiva, se espera que este constituya un modelo para la elaboración de perfiles en las instituciones universitarias y que también se le utilice como herramienta de evaluación de los perfiles ya existentes. 


\section{Refrereiculs}

Alliaud, A. y Vezub, L. (2015). Los saberes docentes en la mira: una aproximación polifónica. Revista de la Escuela de Ciencias de la Educación, (10), 111-130.

Ander-Egg, E. (1992). Técnicas de Investigación Social. Buenos Aires, Argentina: Editorial Magisterio del Río de la Plata.

Annet, K., Sáenz, C., Gonzalo, M., Gorjón, F. y Díaz, C. (2012). Metodología para investigaciones de alto impacto en las ciencias sociales. Madrid, España: Editorial DYKINSON S.L

Ary, D., Jacobs, L.C., Irvine, C.K.S. y Walker, D. (2018). Introduction to research in education. Boston, Estados Unidos: Cengage Learning.

Baeza, P. (2017). Diversidad y Diferenciación en la oferta de programas de doctorado en Chile. Revista Calidad en la Educación, (47), 179-214.

Balaban, C. y Wright, S. (2014). History of Policy Debates about Doctoral Education. UNIKE Notes on Doctoral Education No. 1. Auckland, Nueva Zelanda: Universities in the Knowledge Economy.

Chiappa, R. y Muñoz García, A.L. (2015). Equidad y capital humano avanzado: Análisis sobre las políticas de formación de doctorado en Chile. Psicoperspectivas, 14 (3), 17-30.

Comisión Nacional de Acreditación (2013). Guía para la elaboración del informe de autoevaluación de programas de doctorado. Recuperado de https://www.cnachile.cl/ SiteAssets/Lists/Acreditacion/AllItems/GUIA-PARA-ELABORACION-INFORMEAUTOEVALUACI\%C3\%93N-DOCTORADO.pdf

Comisión Nacional de Acreditación (2016). Propuesta Comité Consultivo de Postgrado: Operacionalización de Criterios de Evaluación para la Acreditación de Programas de Postgrado: Doctorado, Magister Académico y Magister Profesional. Recuperado de https:// www.cnachile.cl/Documentos\%20de\%20Paginas/Operacionalizaci\%C3\%B3n\%20de\%20 criterios $\% 20 \mathrm{de} \% 20$ Postgrado.pdf

Comisión Nacional de Acreditación y Ministerio de Educación (2007). CNAP 1997-2007. El modelo chileno de acreditación de la educación superior. Santiago, Chile: Editora e Imprenta Maval.

Corvalán, J., Falabella, A. y Rojas, M. (2011). El doctorado en educación: un ejemplo de desregulación en el campo de la educación superior en Chile. Calidad en la educación, (34), $15-42$.

Elbaz, M. y Helly, D. (2002). Globalización, ciudadanía y multiculturalismo. Granada, España: Maristán.

Espinoza, O. y González, L. E. (2009). Los estudios de posgrado en Chile: Diagnóstico y proyecciones. Revista Latinoamericana de Estudios Educativos, 39 (3-4), 185-200.

Fernández Fastuca, L. y Wainerman, C. (2015). La dirección de tesis de doctorado: ¿una práctica pedagógica? Perfiles educativos, 37 (148), 156-171.

González, H. y Jiménez, A. (2014). Inserción Laboral de Nuevos Investigadores con Grado de Doctor en Chile. Journal of Technology Management \& Innovation, 9 (4), 132-148.

Hawes, B. y Troncoso, K. (2012). El perfil de egreso. Departamento de Educación en Ciencias de la Salud. Facultad de Medicina, Universidad de Chile, 18, 1-18.

Horgué, C. (2012). La ordenación del Doctorado. Revista de administración pública, (189), 365401.

Jiménez Ramírez, M. (2017b). Convergencia europea e internacionalización del Doctorado: panorámica sobre las tendencias de cambio en España. Estudios pedagógicos, 43(1), 405-422.

Jiménez-Ramírez, M. (2017a). Los nuevos estudios de doctorado en España: avances y retos para su convergencia con Europa. Revista Iberoamericana de Educación Superior, 8 (21), 123137.

Knust, R. y Gómez, S. (2009). La evaluación con enfoque por competencias: ¿Se implementa realmente la evaluación por competencias? Experiencias en Holanda y diferentes países de 
América Latina. Revista Electrónica de Desarrollo de Competencias (REDEC), 3 (1), 104125.

Maura, V. G. y Tirados, R. M. G. (2008). Competencias genéricas y formación profesional: un análisis desde la docencia universitaria. Revista iberoamericana de educación, 47, 185-209.

Ministerio de Educación (2018). Base de datos Matrícula Histórica- Informe SIIES. Santiago, Chile: Ministerio de Educación.

Möller, I. y Gómez, H. (2014). Coherencia entre perfiles de egreso e instrumentos de evaluación en carreras de educación básica en chile. Calidad en la Educación, 41, 17-49.

Namakforoosh, M. (2000) El proceso de investigación. Ciudad de México, México: Prentice-Hall Hispanoamericana

Nebot, I. J. (2009). Introducción. El desafío de los programas de doctorado. En Agencia Nacional de Evaluación de la Calidad y Acreditación (Ed.) El doctorado: logros y desafios (pp. 11-20). Madrid, España: ANECA LIBRO.

Núñez Valdés, K. (2017). Evaluación de los aprendizajes sobre ciudadanía: meta evaluación de los instrumentos utilizados en el segundo ciclo básico chileno. Estudios pedagógicos, 43 (2), 253-276.

Padgett, D. K. (2016). Qualitative methods in social work research. Nueva York, Estados Unidos: Sage Publications.

Padua, J. (2018). Técnicas de investigación aplicadas a las ciencias sociales. Ciudad de México, México: Fondo de Cultura Económica.

Piñuel, J. (2002) Epistemología, metodología y técnicas del análisis de contenido. Galicia, España: Estudios de Sociolingüística 3.

Rivas, L. (2011). Las nueve competencias de un investigador. Revista Investigación Administrativa, 108, 34-54.

Scott, J. W. (2001). Experiência. Revista de estudios de género: La ventana, 2 (13), $42-74$.

Spronken-Smith, R., Brown, K. y Mirosa, R. (2018). Employability and graduate attributes of doctoral graduates. En Spaces, journeys and new horizons for postgraduate supervisión, (pp. 235-256) Stellenbosch, Sudáfrica: African Sun Media

Vera, P. (2010). Historia y estado actual de la formación doctoral en Chile. Revista Digital Universitaria. Recuperado de http://www.revista.unam.mx/vol.11/num5/art47/index.html

Verdejo, P. (2008). Modelo para la Educación y Evaluación por Competencias (MECO). En Propuestas y acciones universitarias para la transformación de la educación superior en América Latina. Informe final del Proyecto 6x4 UEALC (pp. 155-195) Bogotá, Colombia: ASCUN

Villa, A. y Poblete, M. (2007). Aprendizaje basado en competencias. Una propuesta para la evaluación de las competencias genéricas. Bilbao, España: Ediciones Mensajero.

Villarroel, V. y Bruna, D. (2014). Reflexiones en torno a las competencias genéricas en educación superior: Un desafío pendiente. Psicoperspectivas, 13(1), 22-34. 